Historic, Archive Document

Do not assume content reflects current scientific knowledge, policies, or practices. 



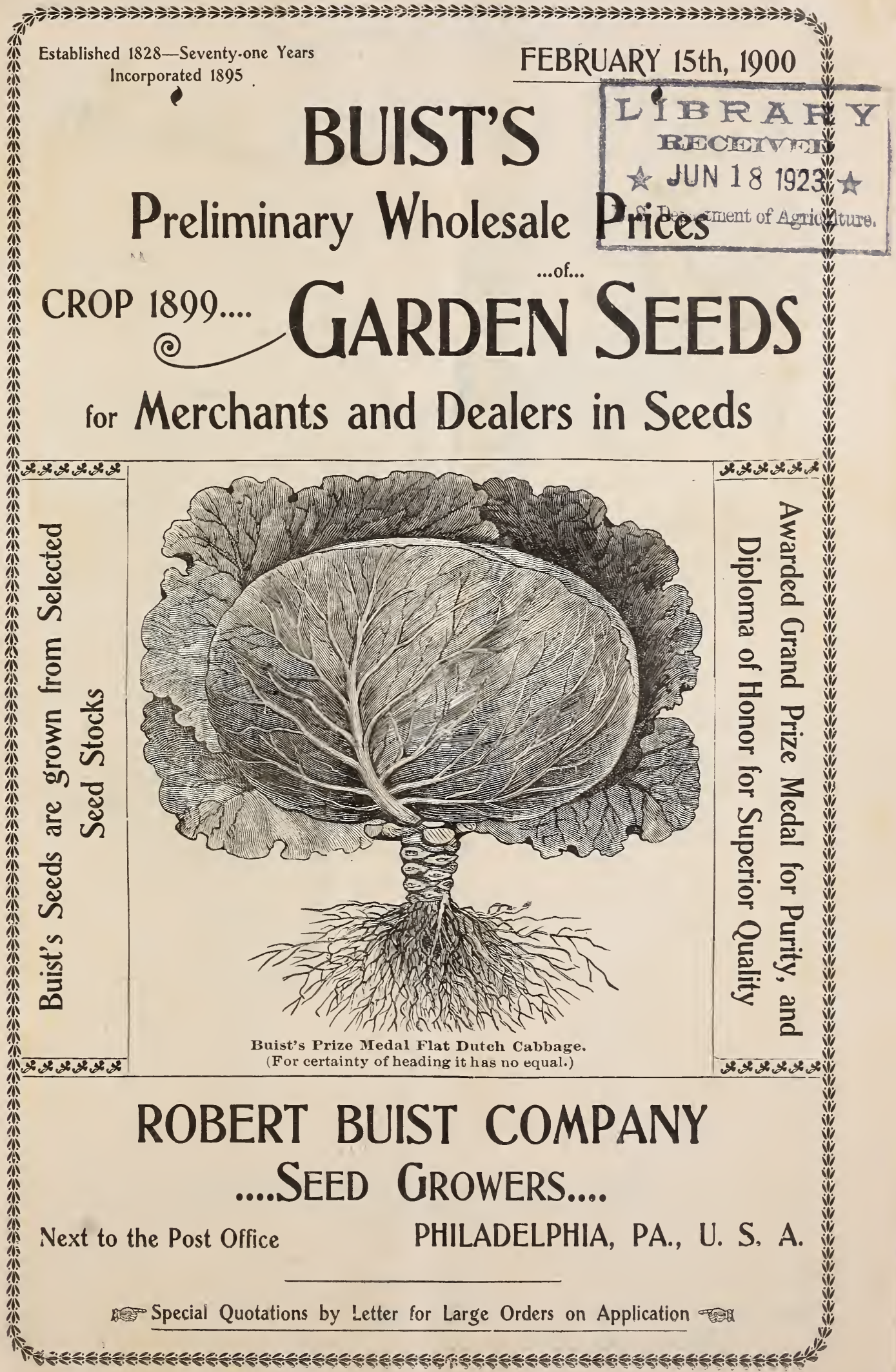




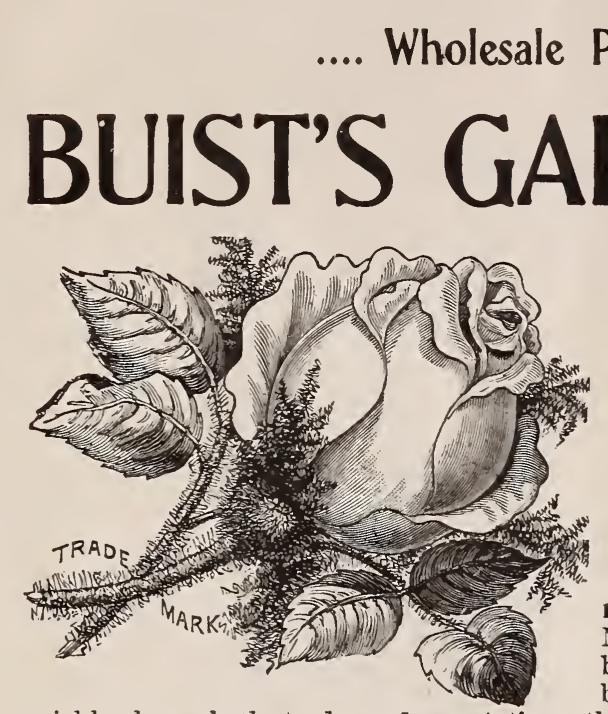

Price Current ....

Grown by us from Selected Seed Stocks.

Are celebrated for their Purity, Fine Quality, Strong Growth.

We sell 10 pounds or over at 100 pound quototion.

Your Garden Seed trade can be very much increased by handling Buist's Prize Medal Seeds ; they are not only the most Popular, but the most Salable. No profit is ever made by the merchant in selling cheap seeds, as he quickly loses both trade and reputation-they are either old stocks or those of an inferior quality. The market is overflowing with old bankrupt stocks, some of which are as old as our grandfather's clock; they are good enough to burn, but not to sow.

Owing to the scarcity of many varieties this season these quotations are only for prompt orders, which we will book now and ship at any date desired. If you are a large purchaser of seeds, it will afford us great pleasure to make you special quotations by letter if you will kindly enclose us a list of your requirements.

\section{BUSH BEANS-Green-Podded.}

\section{CROP VERY SHORT.}

Improved Extra Early Red Speckled Valentine Buist's Lightning Extra Early Valentine Early Mohawk, Six Weeks

Early Long Yellow, Six Weeks

Refuge, or Brown Valentine

Extra Early Refuge

Best of All

Best of All (Buist's Selected Stock)

Shipper's Favorite

Burpee's Stringless Green Pod

Early China Red-Eye

Early White Valentine

White Marrow

White Kidney .

Henderson's Bush Lima

Burpee's Bush Lima
Tel.
Cipher.

Ballot

Babel

Bad

Bay

Beadle

Blood

Band

Bight

Basket

Broker

Bark

Barbaric

Baron

Bedim

Butter

Buoy
10 bush. of
a variety Bush. $\$ 7.75 \$ \$ 8.00$

8.759 .00

$5.25 \quad 5.50$

Sold out

5.75

6.00

6.00

6.25

Sold out

Sold out

Sold out

7.75

8.00

Sold out

Sold out

3.00

3.25

3.00

3.25

5.25

5.50

6.25

6.50

\section{BUSH BEANS.- Wax or Golden-Podded.}

CROP VERY SHORT.

Dwarf German or Black Wax .

Bait

5.25

5.50

Dwarf German Black Wax (Buist's Selected Stock) Bare

5.75

6.00

Improved Dwarf Golden Wax (Rust Proof) . Blank

5.25 
BUSH BEANS-Wax or Golden-Podded-Continued.

Currie's Rust-proof Black Wax .

Dwarf Prolific Black Wax

Dwarf Golden or Cream Wax

Dwarf Golden or Cream Wax (Buist's Selected)

Wardwell's Speckled Kidney Wax

Dwarf Crimson Wax Flageolet

Dwarf Scarlet Market Garden Wax

Dwarf Butter Wax Bismarck.

Dwarf White Wax

Yosemite Mammoth Dwarf Wax

Keeney's Rustless Golden Wax .

Valentine Wax

Refugee Wax

Detroit Wax .

Perfection Wax.

\section{BEANS.-Pole.}

\section{Large White Lima}

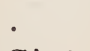

Large White Lima (Extra Size)

Salem Mammoth Lima

\section{Extra Early Jersey Lima}

King of the Garden Lima

Ford's Mammoth Podded Lima

Carolina, Sieva, or Small Lima.

Tel.
Cipher.

10 bush. of a variety.

Brunt

$\$ 6.50$

Per

Bedrench 5.25

$\$ 6.75$

Bake

5.25

$5 \div 0$

Buzz

5.75

Bluff

7.25

5. 50

Bluff

6.25

6.00

7.50

Brisk

6.25

6.50

Border

575

6.50

Bugle

6.00

Bald

5.7.5

6.00

Becoming 7.25

7.50

Bellows

5.75

6.00

Below

Sold out

Beside

6.25

6.50

Benzine

5.25

Binder

5.75

5.50

6.00

Bedeck

5.25

5.50

Boggle

Bray

575

6.00

5.75

6.00

Beholden

6.00

625

Binnacle

5.75

6.00

Bismuth

6.25

6.50

Brain

Bode

Sold out

Dreer's Improved or Challenger Lima

German, or Black Wax Pole

6.25

6.50

Brawl

Banquet

Blow

Improved Large Podded Southern Prolific .

Kentucky Wonder, or Old Homestead .

Southern Crease-Back, or Fat Horse .

Golden Cluster Wax Pole

Birdlime

Battle

Banjo

Brier

Brave

Brand

Bright

Bridle

Sold out

Sold out

Sold out.

Golden Wax Flageolet Pole

8.75

9.00

Sold out

Sold out

Sold out

Sold out

Horticultural, or Wren's Egg

5.25

5.50

Sold out

White Dutch Case Knife

Red Speckled Cut-Short or Corn Hill
Broth
5. 00

4.75

6.00

\section{PEAS.}

The Buist Morning Star Peas, which we introduced a few years since is the earliest and most productive variety known For protection to ourselves and to insure the purchaser true stock, we sell them only in our Sealed Packages For protection to ourselves and to insure the purchaser true stock, we sell them only in our Sealed Packages
and Lead Sealed Sacks, each Seal is stamped Buist. The latter are of green muslin and contain $1 / 8,1 / 4,1 / 2$. or one bushel each. Its great popularity has caused almost ever dealer to catalogue them and a cheap grade of Extra Earlies is substituted. Observe, uuless they are put up in green sacks and sealed with our name they are spurious.

Buist's Extra Early

Pate

2.75

3.00

Buist's Premier Extra Early (Sealed)

Pack

3.00

3.25 
PEAS.-Continued.

Tel.

10 bush of Per

Buist's Early Morning Star (In green sacks, sealed) Pathos

$\$ 3.25 \$ 3.50$

First and Best (New York Stock)

Pang

2.50

2.60

Buist's First and Best

Page

2.60

2.75

Extra Early Market

Park

2.60

2.75

Extra Early Hancock

Pass

2.50

2.60

Extra Early Dandy

Prefer

2.50

2.60

First of All

Paint

250

2.60

Early Alaska

Portly

4.00

Philadelphia Extra Early

Pail

2.50

4.25

Rural New Yorker

Purvey

2.50

2.60

Blue Beauty

Purloin

Improved Early Dan O'Rourke .

Pave

Crop failed

Improved Early Dan O'Rourke (Selected Strain) Pounce

240

2.50

Early Washington

Pawn

2.50

2.60

Early Frame

Peace

2.40

.250

Early May .

Pearl

Admiral .

Pouch

2.40

2.50

French Canner

Petal

2.40

2.50

2.75

3.00

Extra Early Premium Gem

Poise

2.75

McLean's Little Gem .

Plod

3.25

3.00

Horsford's Market Garden

Puff

3.2 .5

3.50

Early American Wonder

Plain

3.25

3.50

Nott's Excelsior

Pencil

3.75

3.50

Champion of England

Prey

400

4.00

Forty Fold

Press

2.50

4.25

Yorkshire Hero .

Pride

2.50

2.75

Early Tom Thumb .

Plate

3.25

2.75

Early Blue Peter

Port

3.25

3.50

Eugenie .

Prank

3.25

3.50

Dwarf Blue Imperial

4.25

3.50

Telephone

Prism

2.50

4.50

Prow

3.50

2.75

Improved Stratagems .

Purple

4.00

3.75

Shropshire Hero

Parish

3.25

4.25

Duke of Albany .

Pastel

3.75

3.50

Heroine.

Prefix

3.00

4.00

Gradus

Purity

7.50

3.25

Potion

3.75

8.00

Laxton's Evolution

Punch

4.25

4.00

Pride of the Market

Prussic

4.75

4.50

Improved Pride of the Market

Pause

3.75

5.00

Telegraph (Long Island Mammoth)

Pottage

3.00

4.00

Bliss's Everbearing .

Public

300

3.25

Bliss's A bundance

Plume

2.75

3.25

3.00

McLean's Advancer 
PEAS.-Continued.

Black-eyed Marrowfat (Hand Picked) Black-eyed Marrowfat (Not Hand Picked)

Large White Marrowfat (Hand Picked)

Large White Marrowfat (Not Hand Picked)

Royal Dwarf White Marrow

Improved Sugar Marrow .

Dwarf or Tall Sugar (Edible Pod)

Melting Sugar .

\section{ARTICHOKE.}

Green Globe

Jerusalem Artichoke Roots

\section{ASPARAGUS. \\ ASPARAGUS ROOTS. \\ Agrail \\ Abbey \\ Abide \\ Affirm \\ Alarm \\ Albino \\ Analogy}

Large Purple Top

Conover's Colossal

Barr's Mammoth

The Palmetto .

Conover's Colossal (Two Years Old).

18.00

18.00

22.00

25.00

Per $100 \mathrm{lbs}$.

Per lb,

2.50

1.50

4.50

4.50

2.00

1.75

2.00

1.75

2.00

3.25

3.00

4.25

.20

.20

.25

.28

Barr's Mammoth (Two Years Old)

Per 100. Per 1000

3.00

The Palmetto (Two Years Old)

\section{BEET.-Our Choicest Selected Stock.}

3.50

4.00

Extra Early Turnip or Bassano

Blind

Per 100 lbs.

Per lb.

22.00

.25

Early Red Turnip .

Birch

20.00

Early Dark Blood Turnip (Selected)

Bit

25.00

Extra Early Egyptian Red Turnip.

Boot

22.00

Crosby's Egyptian

Brass

24.00

25.00

Extra Early Egyptian Red Turnip (Buist's Stock) Big

22.00

Early Eclipse Red Turnip.

Bobbin

22.00

Dewing's Early Red Turnip

Bless

22.00

Philadelphia Early Red Turnip .

Bud

Buist's Extra Early Red Turnip

Blot

45.00

.22

.30

.25

.28

Bastian's Early Red Turnip

Boat

22.00

Lentz's Early Red Turnip

Bold

22.00

.30

.25

.25

.25

.50

Edmand's Blood Turnip

Butment 24.00

.25

.25

Early Othello Blood Turnip

Bend

45.00

.26

Columbia or Excelsior

Brigade

25.00

.50

Boxer

25.00

.30

Stinson's Early Red Turnip

Brewery

22.00

.30

Detroit Dark Red Turnip .

Boston

.25

Electric

Booth

21.00

.25

Swiss Chard

22.00

.25 


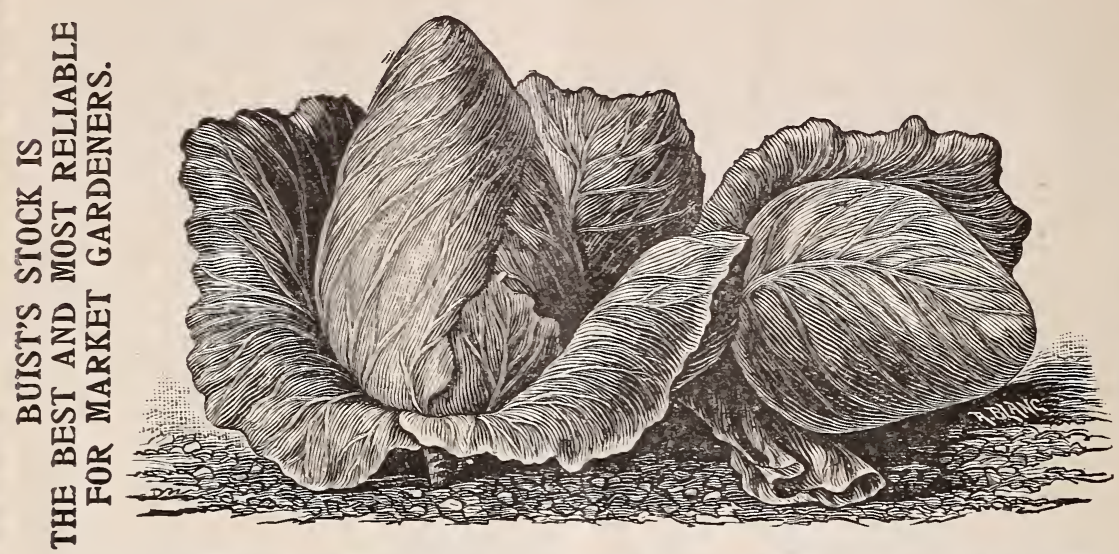

BUIST'S EXTRA EARLY JERSEY WAKEFIELD CABBAGE.

Its Great Features are its Size and Earliness.

BEET.-Our Choicest Selected Stock.-Continued.

Long Blood Red

$\begin{array}{ccc}\text { Tel. } & \text { Per } \\ \text { Cipher. } & 100 \mathrm{lbs} .\end{array}$

Beguile $\$ 18.00$

Buist's Improved Long Blood

Bastian's Half-long Blood .

Beaten $\quad 30.00$

Per lb.

$\$ .20$

.35

Bedaub 22.00

.25

\section{BEET.-Mangolds or Feeding Varieties.}

White French Sugar . . . . . . . Befall

14.00

Lane's Imperial Sugar . . . . . Bias

Giant Long Red Mangel . . . . Beach

15.00

Orange Globe Mangle

Brow

15.00

.18

Champion Mammoth Long Red Mangle

Balm

15.00

.18

Champion Yellow Globe Mangel . . o Brink

15.00

.18

Buist's Mammoth Long Red Mangel . . Bolt

18.00

.18

Buist's Golden Globe Mangel . . . . . Burr

18.00

.20

Golden Tankard Mangel

Chirk Castle Mammoth Red Mangel

Bandage

15.00

.20

.18

Norbiton's Giant Mangel

Bloom

45.00

.50

Brocade

15.00

\section{BROCOLI.}

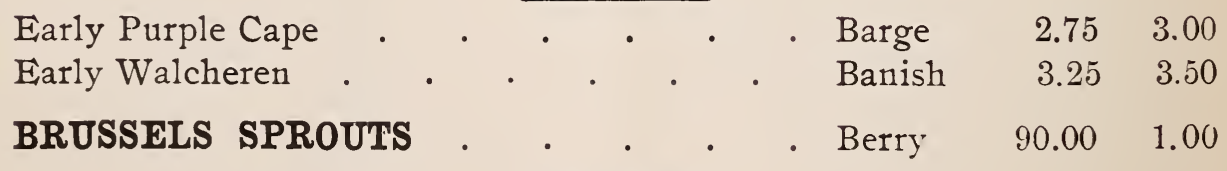

\section{CABBAGE.}

All varieties of Cabbage Seed sold by us are grown from our special seed stocks, which are famous for their great purity and certainty of heading.

Early Dwarf York

Comet $\quad 70.00$

.80

Early Large York

Coy

70.00

.80

Buist's Early Large York

Covert

100.00

1.25

Early Jersey Wakefield (Selected American)

Cynic 240.00

2.50 


\section{THE THREE GREAT VARIETIES OF CABBAGE}

\section{FOR THE SOUTH.}
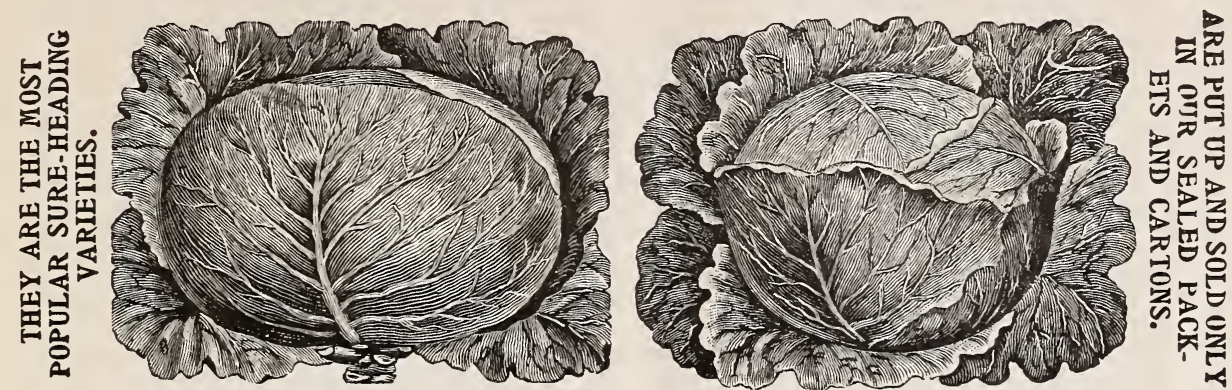

\section{BUIST'S IMPROVED LARGE DRUMHEAD CABBAGE. \\ BUIST'S IMPROVED LARGE FLAT DUTCH. \\ BUIST'S FLORIDA HEADER.}

\section{CABBAGE.-Continued.}

Buist's Extra Early Jersey Wakefield The Charleston, or Large Jersey Wakefield

The Charleston Wakefield (Imported)

Henderson's Early Summer

Early Summer, (Imported)

Henderson's Succession

Early English Winnigstadt

Early French Winnigstadt

Extra Early Express

Early Etampes

Buist's Earliest

All Head Early

Long Island Large Late Drumhead

Long Island Large Late Flat Dutch

Premium Large Late Drumhead (Finest Stock)

Premium Large Late Flat Dutch (Finest Stock)

Large Late Drumhead (Imported).

Large Late Flat Dutch (Imported)

Buist's Improved Late Drumhead (Sealed),

(The Prize Medal Stock) .

Buist's Improved Late Flat Dutch (Sealed),

(The Prize Medal Stock) .

Market Gardeners' Late Flat Dutch, . .

Buist's Florida Header (Sealed), (Prize Stock)

Buist's Short-Stem Brunswick Drumhead

Buist's Early Flat Duch (Sealed)

Buist's Early Drumhead (Sealed)

Improved Early Flat Dutch $\begin{array}{ccc}\text { Tel. } & \text { Per } & \\ \text { Cipher. } & 100 \mathrm{lbs} . \quad \text { Per lb. }\end{array}$

Car $\$ 250.00$

$\$ 2.60$

Caravan 260.00

2.75

Chronic 190.00

2.00

Cancel 135.00

1.50

Clipper 100.00

1.10

Cycle $\quad 175.00$

1.85

Casper $\quad 80.00$

.85

Candle 95.00

1.00

Cartridge 90.00

1.00

Cathedral 70.00

.80

Convey 140.00

1.50

Colony 150.00

1.60

Cruet 115.00

1.25

Cube

115.00

1.25

Cube $\quad 115.00$

1.25

Cup $\quad 115.00$

1.25

Classify $\quad 75.00$

.85

.85

Claimant 75.00

2.00

Creed

175.00

Crop $\quad 175.00$

2.00

Czar

140.00

1.60

Cistern 200.00

2.25

Cast

175.00

2.00

Child

175.00

2.00

Cliff

175.00

2.00 1.40 
CABBAGE.-Continued.

Improved Early Drumhead

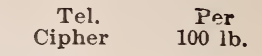

Per ib.

Stein's Early Flat Dutch

Chant $\$ 125.00$

$\$ 1.40$

Early Dwarf Flat Dutch (Imported)

Cascade 140.00

1.50

Early Dwarf Drumhead (Imported)

Louisvile Drumhead

Large Surehead .

Civilian

85.00

.90

Circus

85.00

Crimp

110.00

.90

Cipher 140.00

1.20

All Seasons (American)

Chrome 135.00

1.50

All Seasons (Imported)

Circulate 90.00

1.50

The Lupton

Cosey

150.00

1.00

The Vandergaw .

Chuckle 135.00

1.60

North Carolina Buncombe

Cell

115.00

1.50

Autumn King or World Beater .

Crash

185.00

1.25

Danish Ball Head or Amager .

Commit 185.00

2.00

Short-Stem Drumhead

Hollander or German Export .

Circular 135.00

2.00

Chevalier 185.00

1.40

Fottlers Improved Brunswick

Marblehead Mammoth

Cargo

90.00

2.00

Green Glazed

Crash

110.00

1.00

Ceiling 100.00

$1.2 \tilde{5}$

Red Dutch

Blood Red Berlin

Large Red Drumhead

Mammoth Red Rock .

Improved Red Stone-head

Clamp 110.00

$1.2 \tilde{5}$

1.25

Curt

140.00

1.50

Coliseum 110.00

1.25

Coincide 175.00

2.00

Cuff

160.00

1.75

CABBAGE, SAVOY.

Green Curled Savoy

Cement 135.00

1.50

American Curled Savoy

Censer 110.00

1.25

Green Globe Savoy

Chat

135.00

1.50

Large Drumhead Savoy

Cling $\quad 110.00$

1.25

Perfection Drumhead Savoy

Crisp

110.00

1.25

\section{CARROT.}

Early French Horn

Cincture 40.00

Early Parisian Forcing .

Citadel $\quad 40.00$

.45

Chuck 35.00

Early Scarlet Horn .

Guerande, or Ox-heart

Cogitate

35.00

Connect 30.00

.40

.40

Early Half-long Scarlet (Pointed Root)

Cross

35.00

.35

Early Half-Long Scarlet (Stump Root)

Churn

35.00

Buist's Half-long Scarlet (Pointed Root)

Cymbal $\quad 35.00$

.40

.40

Saint Vallery or Scarlet Intermediate

Codicil

35.00

Half-long Chantenay

Climax $\quad 35.00$

.40

.40

Half-long Nantes

Clarionet 35.00

.40

Half-long Luc

Cruise

35.00 
CARROTS.-Continued.

Half-long Carentan

Tel.

Cipher.

Clergy

Per 100 lbs. Per lb.

Long Orange

Buist's Improved Long Orange .

Comply

$\$ 35.00$

30.00

Canine

35.00

Check

20.00

\section{CAOLIFLOWER.}

Early London

Convey

.50

Chief

Extra Early Paris

Coop

Early Walcheren

Choir

Veitch's Autumn Giant

Chime

.35

Le Normand Short Stem

Church

.50

Large Early White Erfurt .

Copy

1.00

Chimney

1.60

Extra Early Dwarf Erfurt

Buists Prize Early Snowball

Chin

1.60

Crown

.50

4.00

6.00

6.00

3.50

3.00

5.50

14.00

20.00

22.00

5.50

\section{CELERY.}

Large White Solid .

Churl

100.00

1.10

Buist's Large White Solid

Cider

120.00

1.25

Golden Dwarf .

Circle

80.00

Dwarf Golden Heart .

Cape

1.00

Golden Yellow Self-blanching

City

210.00

2.25

Improved White Plume

Corps

100.00

1.10

Contract 110.00

1.25

Giant Pascal

Clap

70.00

.75

Crum

90.00

1.00

Clank

80.00

Sandringham Dwarf White

Classic

90.00

1.00

Kalamazoo Broad Ribbed

Convent 110.00

1.25

Dwarf Pink, or Rose

Colossal 110.00

1.25

Celeriac, or Turnip-rooted Celery

Coral

90.00

1.00

Cosset

10.00

.12

CHERVIL.-Double Curled

Cadge

55.00

.60

COLIARDS. We make the growing of Collard Seed a specialty, and annually sell more seed than any other house in the trade. It is acknowledged that we have the best heading White Sten Cabbage Collards known in the South. The great importance of securing a fine strain is well known by every Southern merchant, as it is one of their most important crops. The cheap Seed offered by the trade is imported from England and is more of a Kale or Wild Cabbage than Collards, and causes great dissatisfaction Georgia (Selected White Stem)

Camus $\quad 50.00$

.60

Southern Blue Stem .

Cadis

50.00

.60

Southern Creole

Callot

55.00

.65

North Carolina Buncombe

Canyon

60.00

.65

Buist's Cabbage Collards

Capoc

70.00

.75

\section{CORN SALAD.}

Large Seeded

Cearack 22.00

.25

Small Seeded .

Crochet

22.00 
CORN.-Garden Varieties.

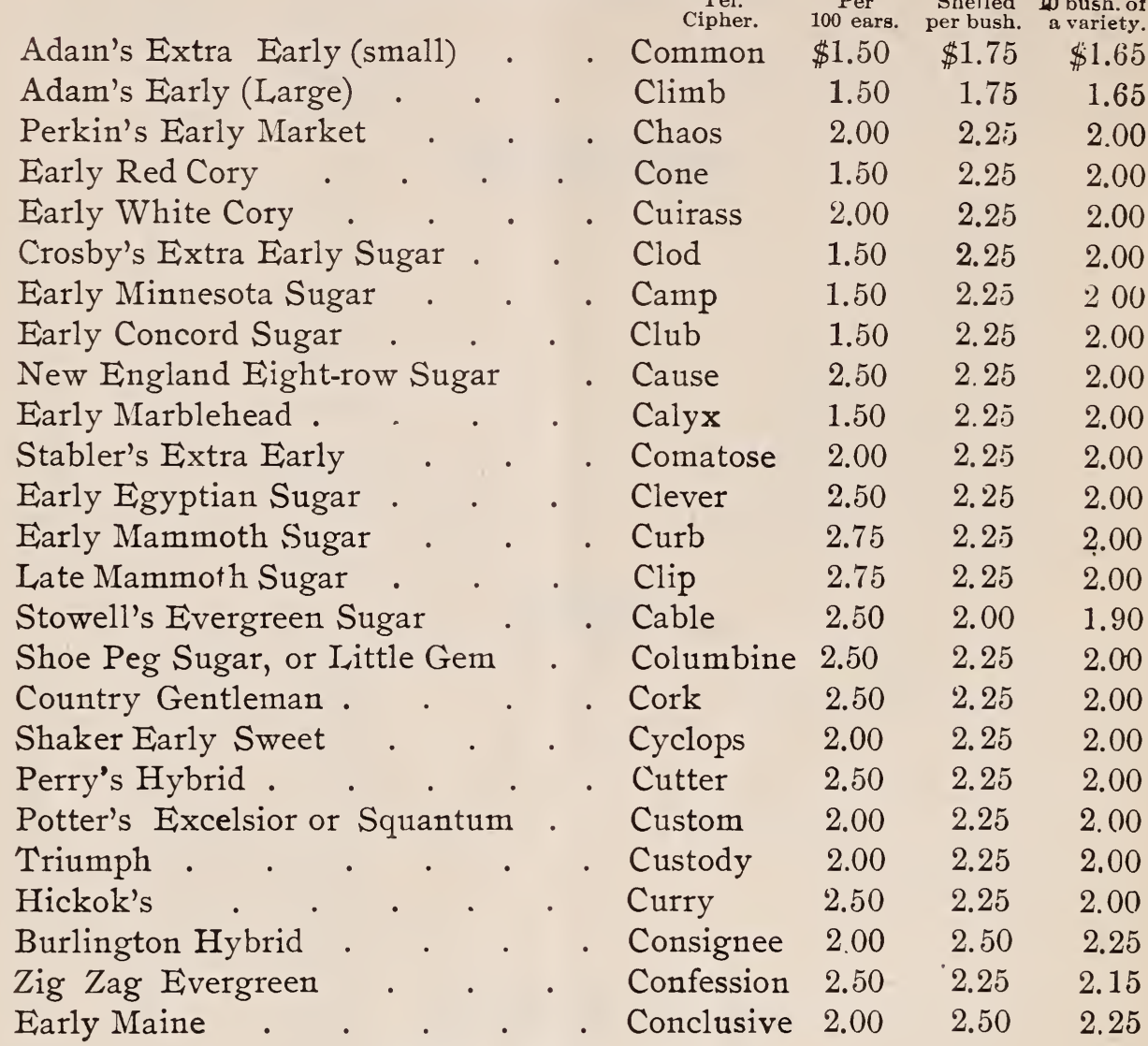

\section{CORN.-Field Varieties.}

All our Shelled Corn is from selected ears, the small points being taken off before shelling.

Common Golded Dent . . . Criminal $1.50 \quad 1.00$

Early Golden Dent (Selected) . . Clef $1.75 \quad 1.10$

$1.75 \quad 1.25$

Buist's Improved Early Golden Dent Cotton

$\begin{array}{ll}1.25 & 1.15\end{array}$

(The best Yellow Field Corn for the South.)

Buist's Prize Medal Southern Snow Flake

(The Best White Field Corn for the South)

Canoe

2.00

1.50

1.40

The above two varieties are only sold in our lead sealed or 2-bushel sacks, each seal stamped Buist.

$\begin{array}{llll}\text { Buist's Large White Flint, or Hominy, Cod } & 2.00 & 1.30 & 1.20\end{array}$

$\begin{array}{lllll}\text { Improved Large Maryland White } & \text { - Comb } & 2.00 & 1.25 & 1.10\end{array}$

$\begin{array}{llllll}\text { White Hickory King ․ . . C Comedian } & \text { I.50 } & 1.25 & 1.20\end{array}$

$\begin{array}{lllll}\text { Improved Early Leaming . . C Crook } & 1.50 & 1.25 & 1.00\end{array}$

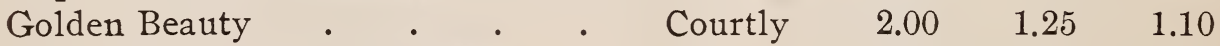

Mammoth Eared Gourd Seed . . Coil $\quad 2.50 \quad 1.25 \quad 1.15$

$\begin{array}{lllll}\text { Chester County Gourd Seed . } \quad \text {. } & \text { Color } & 2.00 & 1.25 & 1.15\end{array}$

Cloud's Early Dent Gourd Seed . Combat $\quad 2.00 \quad 1.25 \quad 1.10$

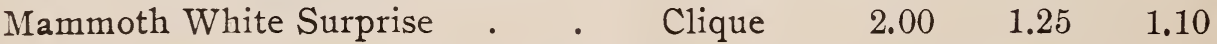




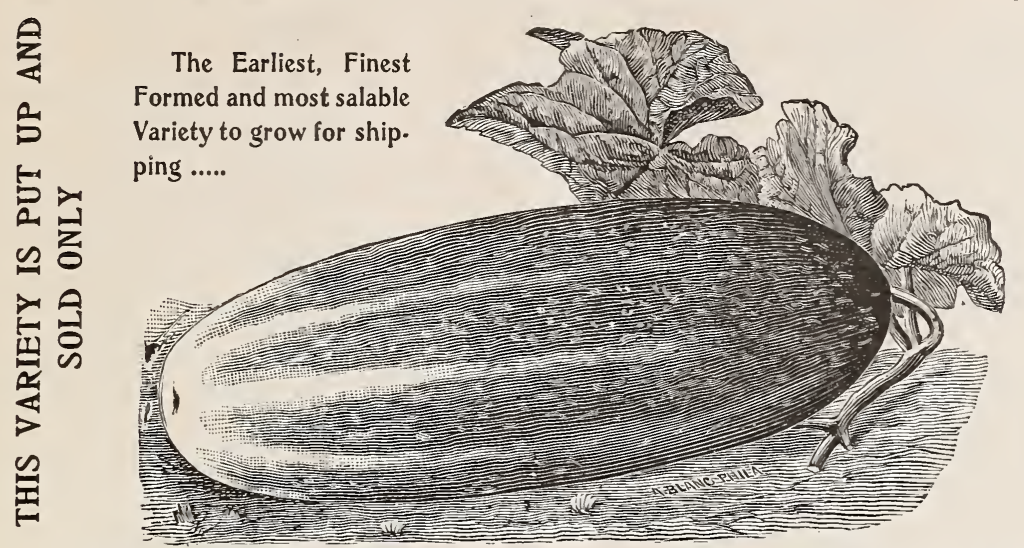

\section{BUIST'S PERFECTION EARLY WHITE SPINE CUCUMBER.}

The Great Market Garden Variety.

CORI

. -Field Varieties.-Continued. Tel.
Cipher.

Per

Shelled 10 bush. of Blunt's White Prolific Clatter 1.50

1.25

1.75

1.25

1.15

Early Yellow Canada Complex

1.75

1.25

Champion White Pearl. . . Curdie

2.00

1.25

Large White Dent

Cupola

1.75

1.25

2.00

1.25

1.15

1.15

1.15

Mastodon Yellow Dent .

Cupel

1.15

1.15

CRESS, or PEP PER GRASS. Tipher.

Per

Curled

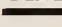

Carbon

25.00

Per lb.

Water

Carlic

275.00

.30

3.00

\section{CUCUMBER.}

Early White Spine

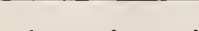

Carmot 22.00

Improved Early White Spine or Arlington

Cartel

28.00

Arlington or Early White Spine (Western Grown.)

Cress

22.00

.30

Buist's Perfection Early White Spine (Sealed),

Evergreen White Spine

Casing

45.00

Cordage

28.00

.50

Evergreen White Spine, (Western Grown.) .

Cupid

22.00

Peerless White Spine

Cracker

28.00

Hill's Forcing White Spine

Console

28.00

.30

Thorburn's Everbearing .

Casque

30.00

.30

Early Frame

Cavern

22.00

.35

Early Cluster.

Caxon

22.00

Early Russian

Census

30.00

.25

Early Short Green

Cephus

22.00

.35

Long Green

Chela

30.00

London L long Green

Cess

30.00

Long Green Turkey

Ceroon

35.00

.25

.35

.35

Buist's Long Green

Canty

45.00

.40

Westerfield's Chicago Pickling . . . . Crosier

22.00 
CUCUMBER.-Continued.

Boston Pickling

New Orleans

Fordhook Pickling .

Livingstone's Evergreen

Giant Pera

Tailby's Hybrid.

Jersey Pickle .

Nichol's Medium Green

Green Prolific Pickle

Gherkin, or Burr

White Wonder

White Pearl

Cool and Crisp

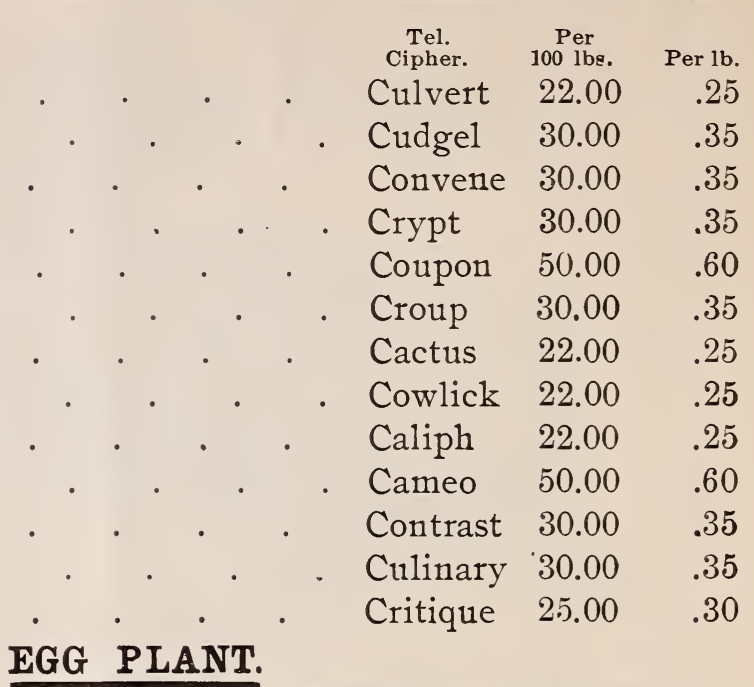

Most of the Egg Plant Seed sold by the trade is of Southern growth, which, owing to poor seed stock, produces imperfect fiuit, both in form and color. The seed which we offer is of our own growth (wo annually grow 25 acres), and there is no finer stock known than the Buist's Improved, which is the best market variety.

New Jersey Improved Large Smooth . ․ . Ease 180.00

Large New York Improved Purple.

Echo

210.00

2.25

Buist's Improved Large Thornless Purple .

Edge

250.00

2.60

(Largest and Finest Fruit and best market variety ; color-dark, rich, purple.)

\section{ENDIVE.}

Mammoth Green Curled .

Eagle

90.00

1.00

Green Curled Winter.

Enrage

70.00

.80

White Curled .

Ebony

70.00

.80

Broad Leaved Batavian

Earl

70.00

.80

Market Gardeners Green Curled

Enigma 120.00

1.25

\section{KALE.}

Dwarf Green Curled Scotch

Kedge

35.00

.40

Philadelphia Green Curled Scotch

Kindred

40.00

Tall Green Curled Scotch

Knight

35.00

.40

Dwarf German Greens

Keel

35.00

Siberian Curled

Kesar

35.00

Knack

35.00

.40

Norfolk Curled .

Knel

60.00

New Intermediate

Kimbo

60.00

Mosbach Curled Winter

New Dwarf Erfurt .

Ken

60.00

Dwarf Curled Berlin .

Knout

60.00

Spring Kale or Sprouts

Kernel

10.00

\section{KOHL RABI.}

Large Green, or White

Key

90.00

1.00

Early White Vienna

Earliest Green Vienna (Short-leaved)

Khan

125.00

1.40

Kirtle $\quad 140.00$

1.50

Early Purple Vienna

Kieve $\quad 150.00$ 
London Broad Flag

LEEK.

\section{LETTUCE.}

Philadelphia Early White Cabbage or Butter

Early Prize Head

Early Curled Silesia

Early Curled Simpson

Black Seeded Simpson

Improved Early Hanson

Eatly Tennisball (White Seeded)

Early Tennisball (Black Seeded)

Buist's Perfection White Forcing

Early Boston Market

Early Speckled Dutch Butter

San Francisco Passion

American Royal Cabbage

Improved Royal Cabbage

White Royal Summer Cabbage .

Black Seeded Royal

Henderson's New York

Philadelphia Market

Big Boston (Selected) .

Premium Cabbage .

The Deacon Cabbage .

Oak Leaved

Denver Market

Golden Standwell

Stubborn Head.

Black Seeded Butter

Drumhead Cabbage

Brown Dutch (Black Seed)

Perpignan, or Early Summer

White Paris Cos (Romaine)

California Cream Butter

Salamander

Blonde Blockhead

Golden Stonehead

Iceberg

Silver Bell

Tel.
Cipher.

Lack

Loop

Lad

Lair

Laky

Lamb

Per

$100 \mathrm{lbs}$.

$\$ 55.00$

65.00

90.00

90.00

75.00

90.00

Per lb.

$\$ .60$

.75

1.00

1.00

.85

1.00

Lance

40.00

.50

Lath

40.00

.45

Lasso

40.00

Larva

40.00

.45

Latin

45.00

.45

Lavish

45.00

.50

League

45.00

Lathe

45.00

Levee

125.00

Legal

55.00

Leper

50.00

Link

75.00

Letch

45.00

I,edme

70.00

Lieve

70.00

Lantern

70.00

Latitude

55.00

Limpet

90.00

Laundry

90.00

Lateral

70.00

Larynx

45.00

Legible

75.00

Lenient

50.00

Liberal

90.00

Liturgy

90.00

Lottery

45.00

Lilac

45.00

Limbo

60.00

Limit

70.00

Lintel

Lisp

55.00

Loathe

50.00

.50

.50

.50

1.40

.60

.55

.80

.50

.75

.75

.75

.60

1.00

1.00

.75

.50

.80

.60

1.0)

1.00

.50

.50

.70

.75

.60

.55

.60

Lengthy 55.00

.60

Ladle

55.00

Lignite

55.00

.60

.60

Lowery

55.00

.60

Lumber 45.00

.50

\section{MELON.-Cantaloupe or Musk.}

Netted Green Citron . $\cdot$. $\cdot$. . Mace

30.00

Madge $\quad 35.00$ 


\section{MELON.-Cantaloupe or Musk.-Continued.}

Rocky Ford

Netted Nutmeg

Chicago Market Nutmeg

Hackensack, or Turk's Cap

Extra Early Hackensack

Miller's Cream, or Osage

Emerald Gem

Acme, or Baltimore Citron

Jersey Belle

Pine Apple

Early Jenny Lind

Golden Netted Gem

Champion Market

Paul Rose

Persian, or Casaba

Bay View

Montreal Market

New Orleans Market

Washington Market

Delmonico .

Banquet

Extra Early Cape May

\section{MELON.-Water.}

Mountain Sweet

Tel.

Cipher. $\quad$ I00 lbs.

Martial $\$ 40.00$

Magic $\quad 30.00$

Per lb.

Minaret 30.00

.35

Myth

$2 \dot{5} .00$

Mattock 3000

.35

.30

Mediate $\quad 30.00$

Meridian 35.00

Meteor $\quad 30.00$

Marsh

30.00

Magnet

30.00

Maim

24.00

Major

4500

Minimum 30.00

.35

.35

Manicure 45.00

Malice

30.00

Mango

30.00

Mantel

30.00

Maple

90.00

Moat

90.00

Metaphor 30.00

Melt

30.00

Money $\quad 30.00$

Kolb's Gem

Striped Gypsy (Selected)

March $\quad 20.00$

Marble 20.00

Mast

25.00

Georgia Rattlesnake (Selected)

Mead

30.00

Duke Jones

Mania

30.00

The Dixie

Motor

24.00

Jordan's Gray Monarch

Model

30.00

Florida Favorite

Manes

20.00

Girardeau's Premium Triumph

Ice Cream (White Seeded)

Ice Cream (Gray Seeded)

Moist

45.00

Merge

24.00

Mé́al

24.00

Phiney's Early

Round Light Icing, or Ice Rind

Monody

24.00

Mica

25.00

Long Light-Icing

Majestic 30.00

Mild

25.00

Seminole

Mink

30.00

Mitre

20.00

Peerless

Pride of Georgia

Moan

24.00

Mode

20.00

Sweet Heart

Monk

25.00

Blue Kolb Gem .

Mank1nd 25.00

Crawford .

Manner

45.00

Mammoth Iron Clad

Merrily

25.00

Black Boulder

Miettle

40.00

Green Preserving Citron

Mock

25.00 


\section{MUSHROOM SPAWN.}

Finest English Milltrack in Bricks 500 lbs. and over at $6 \mathrm{cts}$. per $1 \mathrm{~b}$.

$$
\begin{aligned}
& \text { Tel. } \\
& \text { Cipher. }
\end{aligned}
$$

$$
\text { Myrrh }
$$

$\$ 7.00$

$\$ .08$

\section{MUSTARD.-For Growing.}

White London Mope

Black or Brown

Moral

Giant Southern Curled

Molar

Giant Southern Creole

Molest

55.00

MUSTARD.-For Flavoring.

White London

Mound

8.00

Black or Brown

Musk

8.00

\section{NASTURTIUM, or Indian Cress.}

Tall Yellow (For Pickling)

Notion 50.00

Dwarf Tom Thumb (Mixed)

Neutral 40.00

Tall Mixed

Near $\quad 30.00$

\section{OKRA or GUMBO.}

Buist's Dwarf Green Prolific.

Oasis

30.00

Perkin's Mammoth Podded

Oatl

30.00

Tall, or Long Green

Obey

20.00

White Velvet

Object

25.00

Dwarf White .

Otter

25.00

Improved Dwarf Prolific

Ocean

20.00

Lady Finger

Ode

20.00

\section{ONION}

Buist's Yellow Globe Danvers

Oven

85.00

.90

Large Yellow Globe Danvers .

Obscure

Large Yellow Round Danvers

Orris

80.00

Philadelphia Yellow Dutch or Strasburg

Oblong

80.00

Large Red Wethersfield

Oblate

85.00

.90

.90

White Portugal

Oxide 190.00

2.00

Philadelphia White, or Silver Skin

Observe 190.00

200

Southport Yellow Globe .

Outwit 9500

1.00

Southport Red Globe .

Osier

180.00

Southport White Globe .

Ochre

180.00

Extra Early Red

Oriole

85.00

1.90

1.90

\section{ONION. - Italian and Bermuda Varieties.}

Giant Red Bermuda

Improved Red Bermuda

Giant White Bermuda

Improved White Bermuda

Mammoth Silver King

Mammoth Yellow Prizetaker

Giant Yellow Rocca

Giant Red Rocca

Giant White Tripoli
Olive

175.00

1.90

2.15

2.15

2.55

1.60

1.25

1.25

1.25

1.30 
ONION.-Italian and Bermuda Varieties.-Continued.

Early White Barletta . . . . . . Orator $\$ 175.00$

Early White Queen

Organ 175.00

Early White Pearl

Offer

175.00

1.85

\section{BUIST'S SELECTED ONION SETS}

We are the most extensive growers and shippers of Onion Sets in the trade, with a reputation of ship. ping the finest samples. They are smaller, more uniform in size, and better cleaned than those of Western growth, and will keep longer without sprouting. We also use $7 / 8$ mesh in cleaning them, while Western and many other growers use an inch, making our sets of much smaller size. The measure we send out is also; much larger than Western shippers supply. We also fan and clean each shipment separately. Onion Sets are net cash and subject to cost for barrels and crates in packing.

These prices are for a measured bushel and not a skimped measure of 321 bs., as given in the West and by some Philadelphia houses. A merchant in ordering onion sets expects a measured bushel and not 32 lbs.

Yellow or Red

Omega

Per Bush.

White or Silver Skin

Orbit

Dark Red Wethersfield

Onyx

2.75

3.00

3.00

\section{PARSLEY.}

Finest Double Curled .

Potash $\quad 30.00$

.35

Emerald or Dwarf Extra Curled . . . Partner

30.00

Panel $\quad 35.00$

Buist's Finest Selected Moss Curled . . . Panel

30.00

Champion Moss Curled

Parse

.40

Extra Dark Moss Curled

Plain, or Single

Prance $\quad 30.00$

.35

Path

Sugar or Hollow Crown

PARSNIP.

Improved Guernsey .

Student

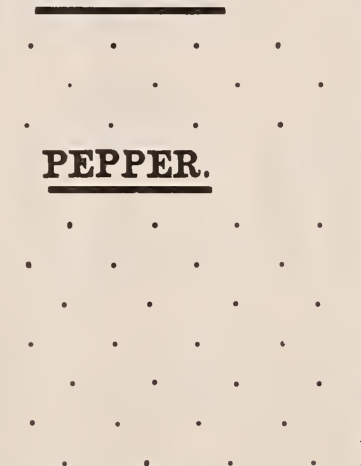

Peak $\quad 18.00$

.20

Prolific

18.00

Peel

20.00

.20

Bell, or Bull Nose

Phaca

125.00

Large Sweet Spanish

Pesky

125.00

Phiz

150.00

1.50

Golden Dawn

Pecan

150.00

1.75

Ruby King

Pendant 125.00

1.75

Squash, or Tomato

Long Red Cayenne

Red Cherry

Phrase 125.00

1.50

1.50

Perna $\quad 150.00$

1.75

\section{PUMPKIN.}

Yellow Cashaw, or Crook-Neck

Pew

30.00

Green Striped Cashaw

Product 30.00

.35

Golden Marrow

Profit

30.00

Pied

30.00

Large Cheese

Purse

50.00

Mammoth King

Puzzle

50.00

Jonathan

Tennessee Sweet Potato

Prelude

30.00

Pursue

30.00

.35

.60

Nantucket

Punt

35.00

Quaker Pie

Pupa

35.00

Pur

12. $(10$ 
Early Long-Scarlet (Short Top)

\section{RADISH.}

Market Gardeners' Long-Scarlet

Long Brightest Scarlet White-Tipped

Early Red Turnip

Early French Scarlet Turnip

Early Deep Scarlet Turnip.

Earliest Erfurt Scarlet Turni

Buist's Earliest Scarlet Button .

Non Plus Ultra Scarlet Turnip

Early Scarlet Turnip (WhiteTipped)

Early Scarlet French Breakfast

Early French Scarlet, or Cardinal Globe

Early Prussian Scarlet Globe (Buist's Stock).

Wood's Early Frame

Early Deep Scarlet Olive-Shaped

French Half-Long Deep Scarlet

Extra Early White Turnip

Early White Turnip

Philadelphia Early White Box

Charter, or Shepherd

Improved Yellow Summer Turnip

Improved White Summer Turnip

Golden Globe Summer

Long Black Spanish (Winter)

Round Black Spanish (Winter)

Chinese Rose (Winter)

White Summer Strasburg

Long White Vienna, or Lady Finger

Buist's Long White

Celestial, or New White Chinese
Buist's Lightning Scarlet Turnip

Tel.
Cipher.

Per

$100 \mathrm{lbs}$.

Race $\$ 22.00$

Regnant 25.00

Rhyme 30.00

Raise

22.00

Review 30.c0

Range

30.00

Ransom

30.00

Relapse

35.00

Road

35.00

Russet

35.00

Rasp

2500

Ratio

25.00

Rout

30.00

Rifle

60.00

Rejoice

22.00

Raven

25.00

Ravine

30.00

Reach

25.00

Realm

22.00

Roster

25.00

Rugged

22.00

Rebuke

30.00

Recede

30.00

Record

30.00

Reign

24.00

Rector

25.00

Reduce

24.00

Ruby

25.00

Revoke

25.00

Relic

35.00

Rescue

35.00

RHUBARB.

Large Victoria

Linaeus.

Rural

75.00

75.00

.80

.80

SALSIFY, or Oyster Plant.

Long White American

Serve

70.00

.80

Mammoth Sandwich Island

Saxon

75.00

.80

Buist's Mammoth White

Sage

90.00

1.00

\section{SPINACH.}

Buist's Perfection Curled (Finest Curled)

Scoff

16.00

Salam

16.00

Saline

16.00

Sally

16.00

.18

Sapid

16.00

.18

Sash

18.00 
SPINACH.-Continued.

Enkhuizen Long Standing

Monstrous Viroflay (Thick-Leaved)

Prickly Seeded

New Victoria

\section{SQUASH.}

Early White Bush, or Patty-Pan

Extra Early White Bush

Mammoth White Bush

Early Golden Bush

Yellow Summer Crook-Neck

Giant Yellow Summer Crook-Neck

Boston Marrow

Buist's Improved Marrow

Hubbard

Chicago Warted Hubbard

Marblehead

London Vegetable Marrow

Fordhook

Mammoth Chili

Orange Marrow

White Pineapple

The Faxon
Tel.

Cipher.

Per

I00 lbs.

Scale $\$ 16.00$

Studio $\quad 16.00$

Scan

18.00

Savor

18.00

Per lb.

$\$ .18$

.18

.20

.20

Scarce

22.00

Scion

25.00

Stroll

25.00

Science

25.00

Scrap

25.00

Sharp

30.00

Sedan

25.00

Scope

45.00

Script

30.00

Scramble 4000

Shoal

30.00

Spectrum 45.00

Spherics 55.00

Sponsal 80.00

Shirk

25.00

Squadron 25.00

Scrag

30.00

The growing of the Tomato espectally for seed, is one of our specialties. Our stocks are unequalled for their great purity and fine quality, which is the result of making an annual selection from which we grow our seed.

Buist's Beauty \} These two varieties are unequalled for shipping, Tendon 185.00

Buist's Belle $\} \quad$ canning, or private growing. Tocsin 225.00

2.00

Buist's Selected Trophy

Teak 150.00

2.50

Buist's Selected Early Acme

Tamp 150.00

1.60

Buist's Selected Paragon

Tardy

150.00

Livingstone's Perfection

Temple 110.00

1.60

1.60

Iivingstone's Favorite

Trench 110.00

1.25

Livingstone's Beauty .

Livingstone's Stone

Tangent 110.00

1.25

Tempt 125.00

1.25

1.40

Early Acme

Tame $\quad 110.00$

1.25

Paragon

Tape $\quad 110.00$

1.25

The Queen .

Trophy

Tawny 110.00

1.25

Taunt 125.00

1.40

The Matchless

Tear

150.00

Mikado

Trait

125.00

1.75

Essex Hybrid

T'rain

110.00

1.50

Cumberland Triumph

Early Ruby

Tease

110.00

1.25

Tint

125.00

1.25

Dwarf Champion

Tower

125.00

1.50

Atlantic Prize

Thesis

110.00

1.50

Ponderosa

Tierce 225.00

1.25

Trucker's Favorite

lendril 175.00 
TOMATO.-Continued.

Golden Trophy

Tel.

Cipher.

Tenor $\$ 140.00$

Per

er lb.

Large Yellow

Red Pear-shaped

Yellow Plum-shaped

Buist's Majestic (This is the largest and finest Tomato in cultivation).

Tepid 140.00

Terse 15000

Text 150.00
$\$ 1.50$

1.50

1.75

1.75

In packets only, per dozen pkts., $\$ 1.00$; per hundred pkts., $\$ 7.00$.

\section{TURNIP.-White Varieties.}

We are very extensive growers of Turnip Seeds, it being one of our specialties, and can supply large orders at lower prices than any house in the trade. Our seed is grown from selected and transplanted roots and is celebrated for its great purity and fine quality.

Red, or Purple Top Flat

Red, or Purple Top (Strap-I.eaved)

Theme $\quad 18.00$

.20

Early White Flat Futch .

Thick $\quad 18.00$

.20

Early White Flat Dutch (Strap-Leaved)

Thirst

18.00

.20

Red, or Purple Top White Globe

Thorn

18.00

.20

Buist's Mammoth Purple Top Globe .

Thread 18.00

.20

Large White Globe

Thrift 20.00

.22

Large. White Norfolk .

Throb $\quad 16.00$

.18

Pomeranean White Globe

Throng 16.00

.18

Southern Snow White Globe

Tide

16.00

.18

Early White Egg

Timid

20.00

Tinge

18.00

.22

Large Cow Horn

Tinsel

20.00

.20

Large White Hanover

Toast

20.00

.22

Sweet German

Token

50.00

.22

Southern Seven Top (For Winter Greens)

Tonic

18.00

.22

Southern Prize (For Turnip or Winter Greens), Torch

20.00

.20

Dixie Land (For Turnip or Winter Greens) . Turn

20.00

Extra Early Red Top Milan

Topaz

30.00

\section{TURNIP.-Yellow Varieties.}

Purple Top Yellow Aberdeen .

Trace $\quad 18.00$

Yellow Stone

Tray $\quad 18.00$

.20

Golden Ball

Treble

20.00

Large Amber Globe

Trend

20.00

.22

Large Yellow Globe

Tress

20.00

.2 .2

.22

\section{TURNIP, RUTA BAGA.}

Improved American Purple-Top Yellow

Tube $\quad 16.00$

Buist's Improved Purple-Top Yellow

Tweed

18.00

Bloomsdale Swede.

Twig

18.00

Improved Long Island Purple-Top Yellow

Twitch

20.00

Sutton's Champion Purple-Top

Tall

16.00

.22

Monarch or Elephant

Tusk

16.00

.18

Budlong Swede

Tenure

30.00

.18

Improved Hardy

Tassel

16.00

Large White, or Russian .

Tyrant

20.00 


\section{Buist's Selected Seed Potatoes,}

\section{Grown . .}

Expressly .

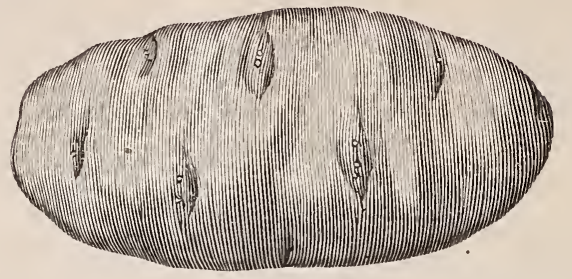

For

Planting .

Packed in large full-sized Barrels or Barrel Sacks, containing 23\%4 Bushels, 165 lbs.

$T \mathrm{H}$

HE Buist Brand of Seed Potatoes have an established reputation throughout the Southern States for their Great Purity, Fine Quality and Productiveness.

They are grown in Maine, Vermont, Nova Scotia and Prince Edward's Island especially for seed, and are of an entirely different quality from the stocks generally sold, which are frequently mixed varieties of a very inferior grade, with the name branded on the barrels to suit the order. These prices are for immediate orders booked with us now, and for shipment during February or March.

Vermont Early Rose

Nova Scotia Early Rose

Prince Edward's Island Rose

Houlton, or Maine Early Rose.

Extra Early Vermont

Early Goodrich

Early Sunrise

Early Snowflake

Burpee's Extra Early

Early Beauty of Hebron

White Star

State of Maine

Bliss' Early Red Triumph, or Imp'd Bermuda

Bliss' Early White Triumph

Pride of the South

Peerless

Carmen No. 3

Burbank's Seedling .

Mammoth Pearl.

Crown Jewell .

Early Ohio

Clark's No. 1,

White Elephant

\section{$25 \mathrm{Bbls}$.}

$\$ 3.00$

3.00

3.00

3.00

3.00

2. 75

2.75

2.75

3.25

2.75

2.75

2.75

3.15

3.15

3.15

2.75

2.75

2.75

2.75

3.25

3.25

3.00

2.75
10 Bbls. Per Bbl.

$\$ 3.15$

3.15

3.15

3.15

3.15

2.85

2.85

2.85

3.35

2.85

2.85

285

3.25

3.25

3.25

2.85

2.85

2.85

2.85

3.35

3.35

3.15

2.85
$\$ 3.25$

3.25

3.25

3.25

3.25

3.00

3.00

3.00

3.50

300

3.00

3.00

3.40

3.40

340

3.00

3.00

3.00

3.00

3.50

3.50

3.25

3.00

Potatoes are sold for net cash; remittance with order or subject to sight-draft on date of shipment. 


\section{Buist's Seeds in Cabinet $=$ Assortments}

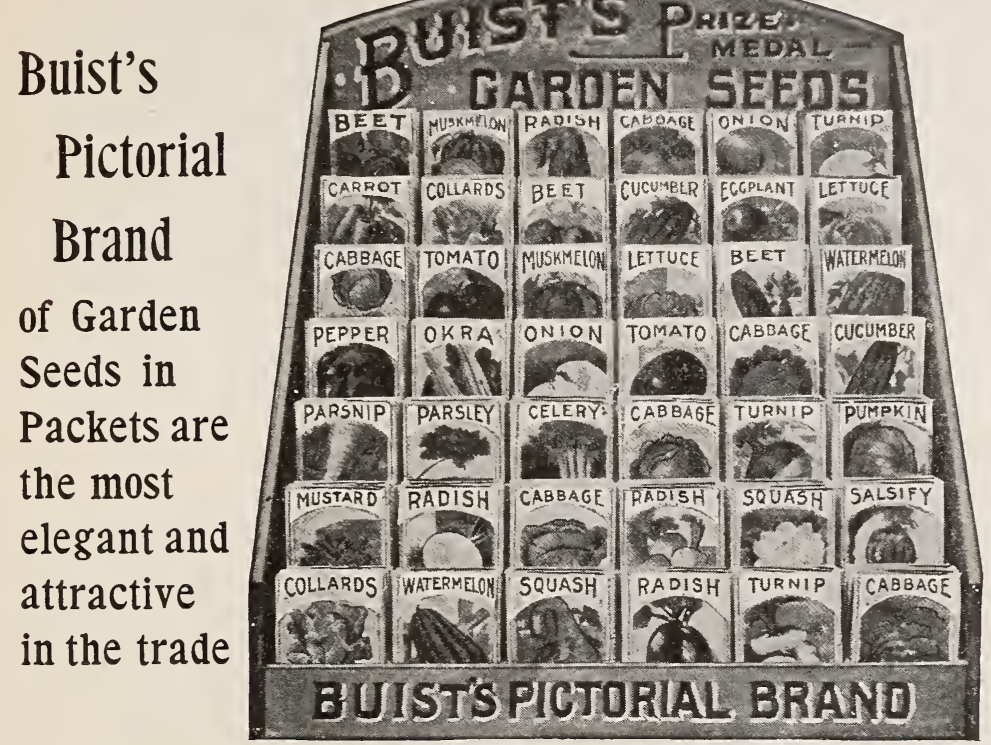

It requires

no talking or persuading to sell them, as they sell themselves. It is a well known fact the vegetables Buist's Seeds produce are as near perfection as can be grown.

\section{CABINET ASSSORTMENTS}

No. 1 contains 500 packets Garden Seeds,

$501 / 3$ pints Peas, Beans and Corn,

20 1/4 Ibs. Turnip Seeds,

\section{Full retail value, $\overline{34.00}$}

Price to Merchants, \$18.00. (Cabinets included.)

No. 2 contains 750 packets Garden Seeds,

75 1/3 pints Peas, Beans and Corn,

$\$ 37.50$

25 1/4 Ibs. Turnip Seeds,

Price to Merchants, \$24.50 $\begin{array}{r}\text { Full retail value, } \\ \text { (Cabinets included.) }\end{array}$

No. 3 contains 1,000 packets Garden Seeds,

$100 \mathrm{1} / 3$ pints Peas, Beans and Corn,

40 1// Ibs. Turnip Seeds,

Full retail value,
Price to Merchants, $\$ 32$ 50. (Cabinets included.)

68.00

No. 4 contains 500 packets Garden Seeds,

$\$ 25.00$

No. $1 / 3$ pints of Peas, Beans or Corn, or $1 / 41 \mathrm{bs}$. Turnip Seeds,

Full retail value are included in this assortment.

Price to Dealer, $\$ 12.00$.

(Cabinets included.)

No. 5 contains 1009 packets Garden Seeds, $\$ 50.00$

No. $1 / 3$ pints of peas, Beans or Corn, or $1 / 4$ lbs. Turnip Seeds, Full retail value are included in this assortment.

Price to Dealers, \$22.00. (Cabinets included.)

BARRELS, BOXES AND SACKS are not returnable for credit, and will only be accepted to be used on future orders. 


\section{Buist's Named Varieties}

...OF...

\section{LARGE FLOWERING SWEET PEAS.}

Alice Eckford ; cream-tinted standard

white wings.

$\$ 0.25$

America ; brightest blood-red striped . .30

Apple Blossom ; bright rosy pink

Aurora ; orange salmon

Blanche Burpee; largest pure white

Blanche Ferry ; pink and white

Blushing Beauty; finest delicate pink

Boreatton; deep maroon

Butterfly; white edged with lavender

Captain of the Blues; bright blueisin purple .

Captivation ; light magenta or claret

Countess of Aberdeen ; shaded pink

Countess of Radnor; pale mauve or lavender

Cardinal; bright scarlet

Cupid Dwarf White ; pure white, grows only six inches high

Cupid Dwarf Pink; rose pink, white wings, six to eight inches high

Dorothy Tennant; deep rosy mauve

Duchess of Edinburgh; scarlet and crimson

Duchess of York; white suffused with pink

Duke of Clarence ; brilliant rosy claret Eliza Eckford; rosy pink and white

Emily Eckford; heliotrope

Emily Henderson; purest white

Empress of India; white and rose

Firefly; brilliant scarlet

Gaiety ; white rose stripe

Gray Friar ; purple white ground

Her Majesty; bright rosy pink .

Ignea; intense scarlet

Indigo King ; blue and purple .

Katharine Tracy ; brilliant pink
Lady Beaconsfield; salmon pink and yellow . . . . . $\quad .30$

Lady Penzance ; pale rose . . .30

Lemon Queen ; blush, tinted lemon . .25

Little Dorrit; pink standard white wings

Lottie Eckford; white suffused with lavender blue

Lovely ; soft shell pink

Mars ; bright scarlet

Meteor ; orange salmon

Miss Hunt ; carmine pink

; deep maroon and dark

Mrs. Jos. Chamberlain ; white striped pale rose

Mrs. Sankey; white

Orange Prince ; orange pink $\quad . \quad .30$

Ovid ; rosy pink

Prima Donna; lovely shade of soft pink

Primrose ; primrose yellow

Princess Beatrice ; carmine rose . .30

Princess May; light lavender . . .30

Princess of Wales; striped mauve on white ground .

Queen Victoria; primrose shaded purple .

Rising Sun ; orange rose and carmine

Royal Robe; delicate pink shaded

Royal Rose ; deep rosy pink

Senator; chocolate striped white

Splendor; rich rose shaded crimson

Stanley; deep maroon

Venus; salmon buff shaded rosy pink

\section{MIXED VARIETIES OF SWEET PEAS.}

Per 1b. Per 100 lb.

Fine Mixed Varieties; $\quad \begin{aligned} & \$ 0.20 \\ & \$ 16.00\end{aligned}$

Choicest Mixed Varieties ; . .25

20.00

Buist's Special Prize Mixture; .35
Eckford's Fine Mixed; . \$0.25

Per $1001 \mathrm{~b}$

Eckford's New Hybrids; .

.35

$\$ 20.00$

French Beauties ; 


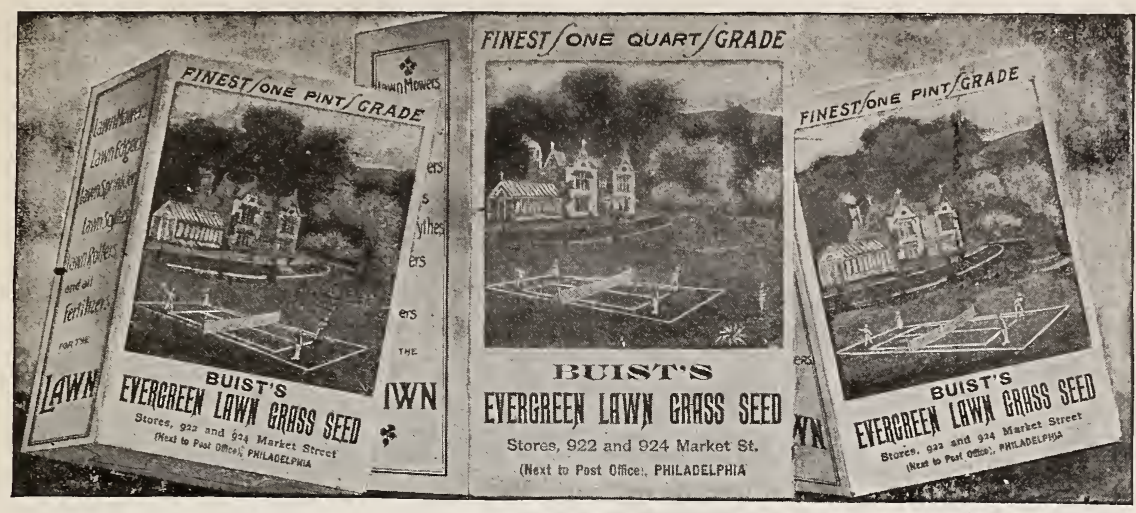

\section{BUIST'S LAWN GRASS SEED}

PUT UP IN CARTONS READY FOR RETAIL TRADE.

IS VERY SALABLE BECAUSE IT GROWS QUICKLY.

Per dozen.

In Pint Cartons which retail at 15 cents each.

1.00

In Quart Cartons which retail at 25 cents each.

1.50

In Two Quart Cartons which retail at 50 cents each.

3.00

In Four Quart Cartons which retail at 75 cents each.

6.00

The Buist's Mixture of Grass Seeds for Lawns, Parks, Tennis and Cricket Grounds has long been famous for producing a succession of verdure throughout the year. It is composed of the finest varieties of grasses, each of which has its season of beauty, and the result of this blending is the producing of a sod which is not only always evergreen and velvety in appearance, but of the color and beauty of an emerald. Many of the is a specialty with our house, which many years of experience has suggested; it requires from 4 to 5 bushels to sow an acre, or for renovating old lawns about half that quantity.

Buist's Evergreen Lawn Mixture (Finest Grade), . " " $\$ 350$

Buist's Evergreen Lawn Mixture (Choice Grade), . . " $\quad 300$

Buist's Eairmount or Central Park Mixture (Finest Grade), " $\quad 300$

Buist's Fairmount or Central Park Mixture (Choice Grade), “ " 250

Buist's Golf Greens Mixture (Finest Grade), • • " 350

VARIETIES OF GRASS SEEDS. I Ibs. $\underset{\text { per }}{\mathrm{Per}}$

Herds, or Red-Top Grass . (Agrostis Vulgaris), . $10 \quad \$ 090$

Kentucky Blue Grass . $\quad$ (Poa pratensis) Extra Cleaned, $\begin{array}{lll}14 & 175\end{array}$

Kentucky Blue Grass ․ (Poapratensis) Fancy Cleaned, $14 \quad 200$

Pennsylvania Green Grass (Poa pratensis) • . . . $14 \quad 225$

Creeping Bent Grass . • (Agrostis stolonifera), . $\quad 12 \quad 250$

Rhode Island Bent Grass $\quad$ (Agrostis Canina), . . $14 \quad 200$

Orchard Grass . . (Dactylis glomerata). . $14 \quad 225$

Timothy . . . . (Phleum pratense), . . $45 \quad 200$

Tall Meadow Oat Grass : (Avena elatior), . . $12 \quad 200$

Yellow Oat Grass . . (Avena flavescens), . . $15 \quad 500$

English Perennial Rye Grass (Lolium perenne), . . $\quad 24 \quad 175$

Italian Rye Grass . • (Lolium Italicum), . . $15 \quad 175$

Johnson Grass . . • . (Sorgum Halapense) . $25 \quad 200$

Sweet Vernal Grass (Anthoxanthemum odoratum), Per 1b.

Meadow Foxtail . . (Alopecurus pratensis), . "

Wood Meadow Grass · (Poa nemoralis), • • • " "

Rough-Stalked Meadow Grass (Poa trivialis), " " 25 


\section{Buist's Garden Seeds \\ IN PACKETS}

\section{The Grand Prize Medal Brand}
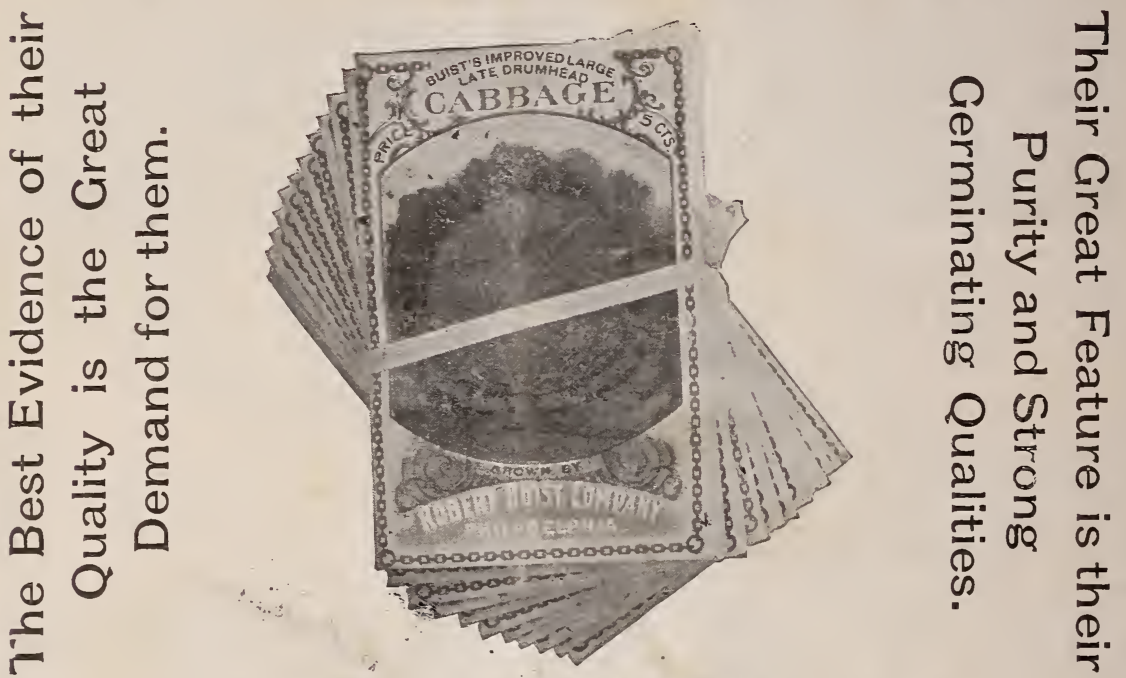

Illustration of Buist's Piotorial Brand Packets.

PRICE FOR THE SMALL OR HALF SIZE PAPERS.

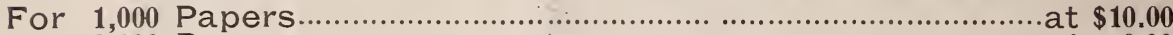

For 3,000 Papers.......................................................................at 9.00

For $\tilde{5}, 000$ Papers................................................................... $\$$

For 10,000 Papers.................................................................. 7.00

For 25,000 Papers.............................................................. 6.50

REDUCED PRICE FOR THE LARGE OR FULL SIZE PAPERS.

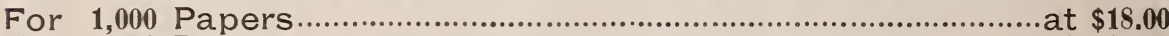

For 3,000 Papers.................................................................at 17.00

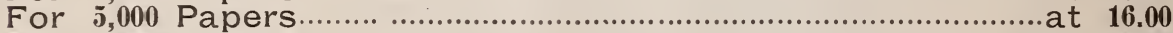

For 10,000 Papers........................................................................at 15.00

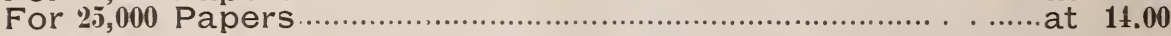

REDUCED PRICE FOR THE BUIST'S NEW PICTORIAL BRAND.

Per, 1,000 Papers.................................................................at $\$ 20.00$

These are the most elegant and attractire Seeds in packets in the trade. Ther sell themselves. They have been reduced this season from $\$ 25.00$ to $\$ 20.00$ per 1,000 packets, and from $\$ 3.00$ to $\$ 2.50$ per 100 .

P. It is to the interest of all merchants who sell seeds to keep Buist's Growth, as no seeds are ever put up in packets but the new crop of each season, those left over from the previous year are cut out whether they grow or not. The result is our seeds will grow equally as well the second year as the first, and many will grow equally as well the third year; such as Beet, Cabbage, Collards. Celery, Cucumber, Kale, Lettuce, Mustard, Parsley, Radish, Pumpkin, Squash, Tomato and Turnip, that is, of course, provided they have been properly kept from heat or dampness.

In the Southern States there are more of them sold than all other hrands combined. They are of new crop each season and grown by us from selected seed stocks. 'There could be nothing finer produced than what we send out.

Terms of Sale.-Four months credit-cash discount, the per cent. if paid within ten days. Accounts are subject to draft when due.

Net Cash Goods are Onion Sets, Potatoes, Bird Seed, Grass, Clorer and all other Agricultural Seeds, and must be remitted for when ordered, or be subject to sight draft on shipment.

Perishable Goods.-Onion Sets and Potatoes are always shipped by us in the very finest order, and packed to carry safely: but as they are subject to damage by excessive heat or cold in transit. over which we have no control, such risk must be assumed by the purchaser. No claims can be allowed for damages.

Barrels, Boxes and Sacks are charged at cost. We deliver all goods F. O. B. Philad'a.

Seed Cabinets.-Display Cabinets made of Ash at $\$ 2.00$ each. Flat Walnut Cabinets at $\$ 2.50$ each. Large Walnut Cabinets containing 49 separate drawers, $\$ 15.00$. These cabinets contain no seeds. 